\title{
ON EQUIVALENCE OF TWO INTEGRABILITY METHODS
}

\author{
H. N. ÖZGEN
}

Received 17 November, 2015

\begin{abstract}
In this paper, we introduce the concept of $|R, p|_{k}, k \geq 1$ integrability of improper integrals and by this definition we prove a theorem, that generalizes a theorem of Orhan [3].
\end{abstract}

2010 Mathematics Subject Classification: 26D15; 40F05; 40D25; 40A10; 35A23

Keywords: Cesàro and Riesz method, improper integrals, divergent integrals, inequalities for integrals

\section{INTRODUCTION}

Throughout this paper we assume that $f$ is a real valued function which is continuous on $[0, \infty)$ and $s(x)=\int_{0}^{x} f(t) d t$. By $\sigma(x)$, we denote the Cesàro mean of $s(x)$. The integral $\int_{0}^{\infty} f(t) d t$ is said to be integrable $|C, 1|_{k}, k \geq 1$, in the sense of Flett [2], if

$$
\int_{0}^{\infty} x^{k-1}\left|\sigma^{\prime}(x)\right|^{k} d x=\int_{0}^{\infty} \frac{|v(x)|^{k}}{x} d x
$$

is convergent. Here, $v(x)=\frac{1}{x} \int_{0}^{x} t f(t) d t$ is called a generator of the integral $\int_{0}^{\infty} f(t) d t$. Let $p$ be a real valued, non-decreasing function on $[0, \infty)$ such that

$$
P(x)=\int_{0}^{x} p(t) d t, p(x) \neq 0, p(0)=0 .
$$

The Riesz mean of $s(x)$ is defined by

$$
\sigma_{p}(x)=\frac{1}{P(x)} \int_{0}^{x} p(t) s(t) d t .
$$

We say that the integral $\int_{0}^{\infty} f(t) d t$ is integrable $|R, p|_{k}, k \geq 1$, if

$$
\int_{0}^{\infty} x^{k-1}\left|\sigma_{p}^{\prime}(x)\right|^{k} d x
$$

is convergent. In the special case if we take $p(x)=1$ for all values of $x$, then $|R, p|_{k}$ integrability reduces to $|C, 1|_{k}$ integrability of improper integrals.

Given any functions $f, g$, it is customary to write $g(x)=O(f(x))$, if there exist $\eta$ 
and $N$, for every $x>N,\left|\frac{g(x)}{f(x)}\right| \leq \eta$.

The difference between $s(x)$ and its $n$th weighted mean $\sigma_{p}(x)$, which is called the weighted Kronecker identity, is given by the identity

$$
s(x)-\sigma_{p}(x)=v_{p}(x),
$$

where

$$
v_{p}(x)=\frac{1}{P(x)} \int_{0}^{x} P(u) f(u) d u .
$$

We note that if we take $p(x)=1$, for all values of $x$ then we have the following identity(see [1])

$$
s(x)-\sigma(x)=v(x) .
$$

Since

$$
\sigma_{p}^{\prime}(x)=\frac{p(x)}{P(x)} v_{p}(x),
$$

condition (1.3) can be rewritten as

$$
s(x)=v_{p}(x)+\int_{0}^{x} \frac{p(u)}{P(u)} v_{p}(u) d u .
$$

In view of the identity (1.4), the function $v_{p}(x)$ is called the generator function of $s(x)$.

Condition (1.1) can also be written as

$$
\int_{0}^{\infty} x^{k-1}\left(\frac{p(x)}{P(x)}\right)^{k}\left|v_{p}(x)\right|^{k} d x
$$

is convergent. We note that for infinite series, an analogous definition was introduced by Orhan [3]. Using this definition, Orhan [3] proved the following theorem dealing with $\left|R, p_{n}\right|_{k}$ and $\left|R, q_{n}\right|_{k}$ summability methods.

Theorem 1. The $\left|R, p_{n}\right|_{k},(k \geq 1)$ summability implies the $\left|R, q_{n}\right|_{k},(k \geq 1)$ summability provided that

$$
\begin{gathered}
n q_{n}=O\left(Q_{n}\right), \\
P_{n}=O\left(n p_{n}\right), \\
Q_{n}=O\left(n q_{n}\right) .
\end{gathered}
$$




\section{MAIN RESUlT}

The aim of this paper is to state Orhan's theorem for $|R, p|_{k}$ and $|R, q|_{k}$ integrability of improper integrals. Now we shall prove the following theorem.

Theorem 2. Let $p$ and $q$ be real valued, non-decreasing functions on $[0, \infty)$ such that as $x \rightarrow \infty$

$$
\begin{aligned}
& x q(x)=O(Q(x)), \\
& P(x)=O(x p(x)), \\
& Q(x)=O(x q(x)) .
\end{aligned}
$$

If $\int_{0}^{\infty} f(t) d t$ is integrable $|R, p|_{k}$, then it is also integrable $|R, q|_{k}, k \geq 1$.

\section{Proof of the Theorem}

Let $\sigma_{p}(x)$ and $\sigma_{q}(x)$ be the functions of $(R, p)$ and $(R, q)$ means of the integral $\int_{0}^{\infty} f(t) d t$. Since $\int_{0}^{\infty} f(t) d t$ is integrable $|R, p|_{k}$, we can write

$$
\int_{0}^{\infty} x^{k-1}\left(\frac{p(x)}{P(x)}\right)^{k}\left|v_{p}(x)\right|^{k} d x
$$

is convergent. Differentiating the equation (1.4), we have

$$
f(x)=v_{p}^{\prime}(x)+\frac{p(x)}{P(x)} v_{p}(x) .
$$

By definition, we obtain

$$
\sigma_{q}(x)=\frac{1}{Q(x)} \int_{0}^{x} q(t) s(t) d t=\frac{1}{Q(x)} \int_{0}^{x}(Q(x)-Q(t)) f(t) d t
$$

and

$$
\begin{aligned}
\sigma_{q}^{\prime}(x) & =\frac{q(x)}{Q^{2}(x)} \int_{0}^{x} Q(t) f(t) d t \\
& =\frac{q(x)}{Q^{2}(x)} \int_{0}^{x} Q(t)\left[v_{p}^{\prime}(t)+\frac{p(t)}{P(t)} v_{p}(t)\right] d t \\
& =\frac{q(x)}{Q^{2}(x)} \int_{0}^{x} Q(t) v_{p}^{\prime}(t) d t+\frac{q(x)}{Q^{2}(x)} \int_{0}^{x} Q(t) \frac{p(t)}{P(t)} v_{p}(t) d t .
\end{aligned}
$$

Integrating by parts of the first statement, we have

$$
\begin{aligned}
\sigma_{q}^{\prime}(x) & =\frac{q(x)}{Q^{2}(x)}\left[Q(x) v_{p}(x)-\int_{0}^{x} q(t) v_{p}(t) d t\right]+\frac{q(x)}{Q^{2}(x)} \int_{0}^{x} Q(t) \frac{p(t)}{P(t)} v_{p}(t) d t \\
& =\frac{q(x)}{Q(x)} v_{p}(x)+\frac{q(x)}{Q^{2}(x)} \int_{0}^{x} Q(t) \frac{p(t)}{P(t)} v_{p}(t) d t-\frac{q(x)}{Q^{2}(x)} \int_{0}^{x} q(t) v_{p}(t) d t \\
& =\sigma_{q, 1}(x)+\sigma_{q, 2}(x)+\sigma_{q, 3}(x), \text { say. }
\end{aligned}
$$


To complete the proof of the theorem, it is sufficient to show that

$$
\int_{0}^{m} x^{k-1}\left|\sigma_{q, r}(x)\right|^{k} d x=O(1) \text { as } m \rightarrow \infty \text {, for } r=1,2,3 \text {. }
$$

Using conditions (2.1) and (2.2), we have

$$
\begin{aligned}
\int_{0}^{m} x^{k-1}\left|\sigma_{q, 1}(x)\right|^{k} d x & =\int_{0}^{m} x^{k-1}\left|\frac{q(x)}{Q(x)} v_{p}(x)\right|^{k} d x \\
& =\int_{0}^{m} x^{k-1}\left(\frac{q(x)}{Q(x)}\right)^{k}\left|v_{p}(x)\right|^{k} d x \\
& =O(1) \int_{0}^{m} x^{k-1}\left(\frac{p(x)}{P(x)}\right)^{k}\left|v_{p}(x)\right|^{k} d x \\
& =O(1) \int_{0}^{m} x^{k-1}\left|\sigma_{p}^{\prime}(x)\right|^{k} d x \\
& =O(1) \text { as } m \rightarrow \infty
\end{aligned}
$$

by virtue of the hypotheses of Theorem 2 .

Applying Hölder's inequality with $k>1$, we get

$$
\begin{aligned}
\int_{0}^{m} x^{k-1} & \left|\sigma_{q, 2}(x)\right|^{k} d x= \\
& =O(1) \int_{0}^{m} x^{k-1}\left(\frac{q(x)}{Q^{2}(x)}\right)^{k}\left(\int_{0}^{x} \frac{Q(t) p(t)}{P(t)}\left|v_{p}(t)\right| d t\right)^{k} d x \\
& =O(1) \int_{0}^{m} \frac{q(x)}{Q^{k+1}(x)}\left(\int_{0}^{x} \frac{Q(t) p(t)}{P(t)}\left|v_{p}(t)\right| d t\right)^{k} d x \\
& =O(1) \int_{0}^{m} \frac{q(x)}{Q^{2}(x)}\left(\int_{0}^{x}\left(\frac{Q(t)}{q(t)}\right)^{k} q(t)\left(\frac{p(t)}{P(t)}\right)^{k}\left|v_{p}(t)\right|^{k} d t\right) \\
& x\left(\frac{1}{Q(x)} \int_{0}^{x} q(t) d t\right)^{k-1} d x \\
& =O(1) \int_{0}^{m} t^{k} q(t)\left(\frac{p(t)}{P(t)}\right)^{k}\left|v_{p}(t)\right|^{k} d t \int_{t}^{m} \frac{q(x)}{Q^{2}(x)} d x \\
& =O(1) \int_{0}^{m} t^{k} \frac{q(t)}{Q(t)}\left(\frac{p(t)}{P(t)}\right)^{k}\left|v_{p}(t)\right|^{k} d t \\
& =O(1) \int_{0}^{m} t^{k-1}\left(\frac{p(t)}{P(t)}\right)^{k}\left|v_{p}(t)\right|^{k} d t \\
& =O(1) \int_{0}^{m} t^{k-1}\left|\sigma_{p}^{\prime}(t)\right|^{k} d t
\end{aligned}
$$




$$
=O(1) \text { as } m \rightarrow \infty
$$

by virtue of the hypotheses of Theorem 2 .

Finally, again by Hölder's inequality with $k>1$, we have

$$
\begin{aligned}
\int_{0}^{m} x^{k-1}\left|\sigma_{q, 3}(x)\right|^{k} d x & =O(1) \int_{0}^{m} x^{k-1}\left(\frac{q(x)}{Q^{2}(x)}\right)^{k}\left(\int_{0}^{x} q(t)\left|v_{p}(t)\right|^{k} d t\right)^{k} d x \\
& =O(1) \int_{0}^{m} \frac{q(x)}{Q^{2}(x)}\left(\int_{0}^{x} q(t)\left|v_{p}(t)\right|^{k} d t\right) \\
& x\left(\frac{1}{Q(x)} \int_{0}^{x} q(t) d t\right)^{k-1} d x \\
& =O(1) \int_{0}^{m} q(t)\left|v_{p}(t)\right|^{k} d t \int_{t}^{m} \frac{q(x)}{Q^{2}(x)} d x \\
& =O(1) \int_{0}^{m} \frac{q(t)}{Q(t)}\left|v_{p}(t)\right|^{k} d t \\
& =O(1) \text { as } m \rightarrow \infty
\end{aligned}
$$

by virtue of the hypotheses of Theorem 2 .

This completes the proof of the theorem.

Theorem 3. Let $p$ and $q$ be real valued, non-decreasing functions on $[0, \infty)$ such that as $x \rightarrow \infty$

$$
\begin{aligned}
& x p(x)=O(P(x)), \\
& Q(x)=O(x q(x)), \\
& P(x)=O(x p(x)) .
\end{aligned}
$$

If $\int_{0}^{\infty} f(t) d t$ is integrable $|R, q|_{k}$, then it is also integrable $|R, p|_{k}, k \geq 1$.

Proof. In Theorem 2 if we take $p(x)=q(x)$ and $q(x)=p(x)$, then we get Theorem 3.

Theorem 4. Let $p$ and $q$ be real valued, non-decreasing functions on $[0, \infty)$ such that as $x \rightarrow \infty$

$$
\begin{aligned}
& x p(x)=O(P(x)), \\
& P(x)=O(x p(x)), \\
& x q(x)=O(Q(x)), \\
& Q(x)=O(x q(x)) .
\end{aligned}
$$

Then the $|R, p|_{k}$ integrability of $\int_{0}^{\infty} f(t) d t$ is equivalent to the $|R, q|_{k}$ integrability of $\int_{0}^{\infty} f(t) d t$, where $k \geq 1$. 


\section{REFERENCES}

[1] I. Çanak and U. Totur, "A tauberian theorem for cesàro summability factors of integrals," Appl. Math. Lett., vol. 24, no. 3, pp. 234-245, 2011.

[2] T. M. Flett, "On an extension of absolute summability and some theorems of littlewood and paley," Proc. London Math. Soc., vol. 7, pp. 113-141, 1957.

[3] C. Orhan, "On equivalence of summability methods," Math. Slovaca., vol. 40, no. 2, pp. 171-175, 1990.

\section{Author's address}

H. N. Özgen

Department of Mathematics, Faculty of Education, Mersin University, TR-33169 Mersin, Turkey E-mail address: nogduk@gmail.com 